\title{
A UNIQUENESS THEOREM FOR A CLASS OF HARMONIC FUNCTIONS
}

\author{
A. J. LOHWATER ${ }^{1}$
}

In this note we shall establish a uniqueness theorem for the class of functions $u(r, \theta)$, harmonic in $|z|<1$, for which

$$
\int_{0}^{2 \pi}|u(r, \theta)| d \theta<M, \quad z=r e^{i \theta},
$$

where $M$ is a finite constant independent of $r$.

Theorem. Let $u(r, \theta)$ be harmonic in $|z|<1$ and there satisfy (1). Let $\lim _{r \rightarrow 1} u(r, \theta)=0$ for almost all $\theta$ in $0 \leqq \theta \leqq 2 \pi$, and let $\lim _{r \rightarrow 1} u(r, \theta)$ $= \pm \infty$ for all $\theta$ belonging to a countable set $E$ in $0 \leqq \theta \leqq 2 \pi$. If $\lim _{r \rightarrow 1}$ $u(r, \theta)$, wherever else it may exist, is not infinite, then there exist real constants $c_{n}$ with $\sum_{n=1}^{\infty}\left|c_{n}\right|<\infty$ such that, in $|z|<1$,

$$
u(r, \theta) \equiv \sum_{n=1}^{\infty} c_{n} K\left(r, \theta-\theta_{n}\right),
$$

where $\bigcup_{n=1}^{\infty} \theta_{n}=E$ and $K(r, \theta-\alpha)=\left(1-r^{2}\right) /\left(1+r^{2}-2 r \cos (\theta-\alpha)\right)$.

A harmonic function satisfying (1) in $|z|<1$ has an integral representation

$$
u(r, \theta)=\frac{1}{2 \pi} \int_{0}^{2 \pi} K(r, \theta-\phi) d \mu(\phi),
$$

where $\mu(\phi)$ is of bounded variation in the interval $0 \leqq \phi \leqq 2 \pi$. We consider the Lebesgue decomposition of $\mu(\phi)$ :

$$
\mu(\phi)=\nu(\phi)+g(\phi)
$$

where $\nu(\phi)$ is absolutely continuous with $\mu^{\prime}(\phi)=\nu^{\prime}(\phi)$ almost everywhere, and $g(\phi)$ is of bounded variation with $g^{\prime}(\phi)=0$ almost everywhere. Now, for any $\phi$ for which $\mu^{\prime}(\phi)$ exists, which is the case almost everywhere, $\lim _{r \rightarrow 1} u(r, \phi)=\mu^{\prime}(\phi)$. Since $\lim _{r \rightarrow 1} u(r, \theta)=0$ almost everywhere, we have $\nu^{\prime}(\phi)=0$ almost everywhere, so that $\nu(\phi)$ in (4) is identically constant. Now, it is known ${ }^{2}$ that, for any point of discontinuity $\theta_{0}$ of $\mu(\phi), \lim _{r \rightarrow 1} u\left(r, \theta_{0}\right)= \pm \infty$. Consequently the points

Presented to the Society, September 7, 1951; received by the editors July 9, 1951.

${ }_{1}^{1}$ The work on this paper was performed under sponsorship of ONR.

${ }^{2} \mathrm{Cf}$. the author's paper The boundary values of a class of meromorphic functions, to appear in Duke Math. J. 
of discontinuity of $\mu(\phi)$ are contained in the set $E$. If we write $g(\phi)$ $=g_{1}(\phi)+\psi(\phi)$, where $g_{1}(\phi)$ is a continuous function of bounded variation with $g_{1}^{\prime}(\phi)=0$ almost everywhere and $\psi(\phi)$ is a step function, it follows from [1, pp. 127-128] that, unless $g_{1}(\phi)$ reduces to a constant, $g^{\prime}(\phi)$ is infinite on a noncountable set of points. This implies that $\lim _{r \rightarrow 1} u(r, \theta)$ is infinite on a noncountable set of values of $\theta$, which is contrary to hypothesis. Hence $g_{1}(\phi)$ is identically constant, and $\mu(\phi)$ reduces to a pure step function.

Since $\mu(\phi)$ in (3) reduces to a step function, we may replace the Stieltjes integral there by a series; more precisely: there exists a sequence of real numbers $\left\{c_{n}\right\}(n=1,2, \ldots)$ with $\sum_{n=1}^{\infty}\left|c_{n}\right|<\infty$ such that

$$
u(r, \theta)=\sum_{n=1}^{\infty} c_{n} K\left(r, \theta-\theta_{n}\right),
$$

and where $2 \pi c_{n}$ represents the saltus of the step function $\psi(\phi)$ at the jump points $\theta_{n}$. Hence the theorem is proved.

The following corollary is an easy consequence of our theorem: Let $u(r, \theta)$ be harmonic in $|z|<1$ and there satisfy (1); let $\lim _{r \rightarrow 1} u(r, \theta)$ $=0$ for almost all $\theta$ in $0 \leqq \theta \leqq 2 \pi$ and let $\lim _{r \rightarrow 1} u(r, \theta)$, wherever else it may exist, not be infinite. Then $u(r, \theta)$ is identically zero in $|z|<1 .^{3}$ Indeed, since $\lim _{r \rightarrow 1} u(r, \theta)$, wherever it exists, cannot be infinite, it follows that all the constants $c_{n}$ in (2) must be zero.

To show that condition (1) is essential we exhibit the function

$$
u(r, \theta)=\frac{\partial}{\partial \theta}\left\{\frac{1-r^{2}}{1+r^{2}-2 r \cos \theta}\right\}=\frac{-2 r\left(1-r^{2}\right) \sin \theta}{\left[1+r^{2}-2 r \cos \theta\right]^{2}}
$$

which is harmonic in $|z|<1$ and has the property that, for all $\theta$, $\lim _{r \rightarrow 1} u(r, \theta)=0$.

\section{BIBLIOGRAPHY}

1. S. Saks, Theory of the integral, Warsaw, 1937.

The University of Michigan

${ }^{3}$ We remark that P. C. Rosenbloom has proved a weaker form of this corollary assuming that $\lim _{r \rightarrow 1} u(r, \theta)=0$ for every value of $\theta$. His result will appear in his forthcoming book on partial differential equations. 